\title{
4-hexylresorcinol stimulates the differentiation of SCC-9 cells through the suppression of E2F2, E2F3 and Sp3 expression and the promotion of Sp1 expression
}

\author{
SEONG-GON KIM ${ }^{1 *}$, AN-SOOK KIM ${ }^{2}$, JAE-HWAN JEONG ${ }^{3}$, JE-YONG CHOI $^{3 *}$ and HAEYONG KWEON ${ }^{4}$ \\ ${ }^{1}$ Department of Oral and Maxillofacial Surgery, College of Dentistry, Gangneung-Wonju National University, \\ Gangneung 210-702; ${ }^{2}$ Department of Dentistry, Hallym University, Chuncheon 200-702; \\ ${ }^{3}$ Department of Biochemistry and Cell Biology, WCU Program, CMRI, Skeletal Diseases \\ Genome Research Center, School of Medicine, Kyungpook National University, Daegu 700-422; \\ ${ }^{4}$ National Academy of Agricultural Science, RDA, Suwon 441-707, Republic of Korea
}

Received February 23, 2012; Accepted May 14, 2012

DOI: $10.3892 /$ or.2012.1845

\begin{abstract}
The dormancy-inducing factors of bacteria inhibit tumor cell growth. In the present study, we evaluated the antitumor effects of the dormancy-inducing factor 4-hexylresorcinol (4-HR) using real-time cell electronic sensing (RT-CES) in SCC-9 cells (tongue squamous cell carcinoma cells). Treatment with 4-HR suppressed the growth of SCC-9 cells in a dosedependent manner. We used a DNA microarray to identify genes that showed a significant change in expression upon 4-HR administration in SCC-9 cells. Among the differentially expressed genes, the protein expression of several cell proliferation related factors, including E2F1, E2F2, E2F3, E2F4, E2F5, E2F6, Sp1 and Sp3, were determined by western blot analyses. Treatment with 4-HR strongly suppressed E2F2 and slightly suppressed E2F3 but did not change the expression of E2F1, E2F4, E2F5 and E2F6 relative to no treatment. Furthermore, 4-HR increased Spl expression in a dose-dependent manner and decreased Sp3 expression. Therefore, the ratio of Sp1 to Sp3, an important driving force of epithelial cell differentiation, was drastically increased. Consistent with this observation, 4-HR increased the expression of the epithelial cell differentiation markers involucrin and keratin 10 . Together, our results indicate that 4-HR induces the differentiation of SCC-9 via the modulation of the E2F-mediated signaling pathway.
\end{abstract}

Correspondence to: Dr Seong-Gon Kim, Department of Oral and Maxillofacial Surgery, College of Dentistry, Gangneung-Wonju National University, Jihyun-dong, Gangneung, Gangwon-do 210-702, Republic of Korea

E-mail: epker@chollian.net

Dr Je-Yong Choi, Department of Biochemistry and Cell Biology, School of Medicine, Kyungpook National University, 101 Dong-In Dong, Jung-Gu, Daegu 700-422, Republic of Korea

E-mail: jechoi@knu.ac.kr

*Contributed equally

Key words: 4-hexylresorcinol, SCC-9 cells, differentiation, E2F

\section{Introduction}

The signaling pathways related to cell differentiation and senescence do not function properly in malignant tumor cells. As a result, tumor cells exhibit uncontrolled and invasive growth. Differentiation therapy is one emerging technique for the treatment of malignant cancer (1). Tumor cells originate from normal cells and many different carcinogenic stimuli can contribute to the development of cancer. Several key genes responsible for regulating apoptosis or senescence may undergo loss of function mutations in various tumors, particularly oral squamous cell carcinoma (OSCC) (2). The main objectives of differentiation therapy may be achieved by the bypassing the mutated pathway, thus tipping the balance from carcinogenesis to terminal differentiation, or rescuing the senescence machinery.

E2F overexpression in squamous cell carcinoma cells results in aberrant proliferation and the suppression of the squamous differentiation program $(3,4)$. E2F transcription factors promote the expression of genes related to DNA synthesis and cell cycle progression, resulting in cell proliferation $(5,6)$. An increase in E2F activity is often associated with inappropriate cell proliferation and/or apoptosis (7), whereas a decrease in E2F activity is generally associated with a reduction in the proliferation capacity of cells (8). The deregulation of E2F activity contributes to carcinogenesis (9). E2Fs are involved in the differentiation of many cell types, such as myocytes (10), megakaryocytes (11), and adipocytes (12). The specific factor (Sp)1 to Sp3 ratio has been recognized as an important driving force of epithelial cell differentiation (1). Dysregulation of the expression of these factors or the change of the relative $\mathrm{Sp} 1$ and $\mathrm{Sp} 3$ expression levels result in dysregulated cell proliferation and differentiation (1). The direct downregulation of E2F and upregulation of Sp1 increases the expression of differentiation specific markers such as transglutaminase-1 and involucrin (1). Therefore, the expression of E2F factors and the ratio of $\mathrm{Sp} 1$ to $\mathrm{Sp} 3$ expression are important parameters determining the epithelial cell proliferation and differentiation.

The non-isoprenoid lipid is found in a wide range of plant and bacterial species. These lipids exert non-specific antioxidant 
and antimutagenic effects, and can regulate cell proliferation (13). Chemical analogs of these lipids have exhibited anticancer effects in animal models of colon (14), lung (15), and pancreatic tumors (16). 4-hexylresorcinol (4-HR) is also non-isoprenoid lipid. According to recent reports, 4-HR inhibits the transglutaminase- 2 and NF- $\mathrm{KB}$ pathways $(17,18)$, and $4-\mathrm{HR}$ also shows a synergistic effect with cisplatin in KB cells $(17,18)$. Interestingly, the cancer cells that survived 4-HR administration were less active and morphologically differentiated than the active proliferating control (data not shown). These findings suggest the possibility that 4-HR may induce differentiation in tumor cells. We hypothesize that 4-HR induces the differentiation of tumor cells through the control of cell cycle-related genes like E2F transcription factors.

The objectives of this study were: i) to evaluate of the effect of 4-HR on E2F signaling pathway on OSCC cells, and ii) to evaluate the level of expression of differentiation markers in OSCC cells after the administration of 4-HR.

\section{Materials and methods}

Cell culture. Cell line SCC-9 cells (human tongue carcinoma; ATCC; Manassas, VA, USA) were maintained in monolayer cultures in DMEM (Invitrogen, Carlsbad, CA, USA) supplemented with $10 \%$ fetal bovine serum containing L-glutamine, vitamins (Life Technologies, Inc., Grand Island, NY, USA), and penicillin-streptomycin (Flow Laboratories, Rockville, MD, USA). The cells were incubated in a mixture of $5 \% \mathrm{CO}_{2}$ and $95 \%$ air at $37^{\circ} \mathrm{C}$. The cultures were maintained for no longer than 12 weeks after recovery from frozen stocks.

Real-time cell electronic sensing (RT-CES). RT-CES is a measurement of impedance in a gold-plated culture dish. Because the cellular membrane is composed of lipid bilayer, increased cellular growth increases the impedance. The labelfree detection of cell growth via RT-CES was conducted using an xCELLigence ${ }^{\circledR}$ system (Roche Applied Science, Penzberg, Germany) and special gold-plated 96-well culture dishes in which the SCC-9 cells were grown. 4-HR was added at a concentration of 1,5 or $10 \mu \mathrm{g} / \mathrm{ml}$ to each well $20 \mathrm{~h}$ following the initial seeding. No 4-HR was added in the control wells. The cell index values derived from the measured impedance were determined every hour for 4 days.

Western blot analysis. Whole cells were lysed in ProteoJET ${ }^{\mathrm{TM}}$ Mammalian Cell Lysis Reagent (Thermo Scientific, Waltham, MA, USA) containing protease inhibitor cocktail (SigmaAldrich Inc., St. Louis, MO, USA). Proteins were separated on $10 \%$ SDS-polyacrylamide gels and transferred to PVDF membranes. Blots were blocked with 5\% skim milk powder in Tris-buffered saline (20 mM Tris- $\mathrm{HCl}, 137 \mathrm{mM} \mathrm{NaCl}, \mathrm{pH}$ 7.6) containing $0.1 \%$ Tween-20 (TBS-T buffer) for $1 \mathrm{~h}$ at room temperature (RT). Western blot analyses were performed with anti-E2F1 (Abcam Inc. Cambridge, MA, USA), anti-E2F2 (Abcam Inc.), anti-E2F3 (Abcam Inc.), anti-E2F4 (Abcam Inc.), anti-E2F5 (Abcam Inc.), anti-E2F6 (Abcam Inc.), antiSp1 (Abcam Inc.), anti-Sp3 (Abcam Inc.), anti-keratin 10 (Santa Cruz Biotechnology, Santa Cruz, CA, USA), anti-involucrin (SantaCruz Biotechnology), and anti- $\beta$-actin (Sigma-Aldrich Inc.) antibodies. Primary antibodies were added to the TBS-T

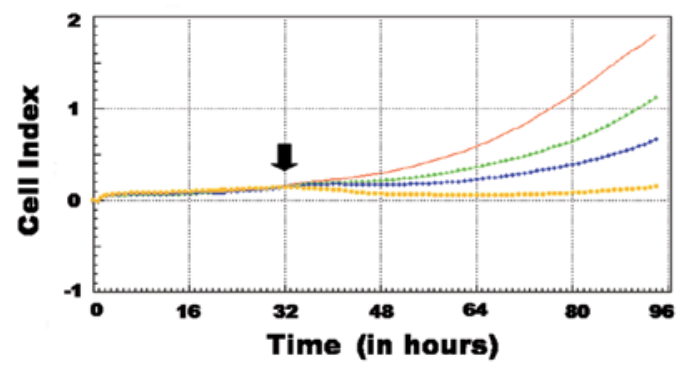

Figure 1. RT-CES-monitored cell growth (red, untreated control; green, $1 \mu \mathrm{g} / \mathrm{ml} 4$-HR; blue, $5 \mu \mathrm{g} / \mathrm{ml} 4$-HR; yellow, $10 \mu \mathrm{g} / \mathrm{ml} 4-\mathrm{HR})$. The arrow at $\sim 32 \mathrm{~h}$ indicates the application of 4-HR to the cultured cells. Treatment with 4-HR inhibited the growth of SCC-9 in a dose-dependent manner.

buffer at a 1:1000 dilution and incubated for 90 min at RT prior to incubation with HRP-conjugated secondary antibodies (1:5000 dilution; Santa Cruz Biotechnology) for $1 \mathrm{~h}$ at room temperature. The proteins were detected using a chemiluminescence detection system (Bio-Rad, Hercules, CA, USA). The relative expression ratio of $\mathrm{Sp} 1$ to $\mathrm{Sp} 3$ was determined using Image Lab software (Bio-Rad).

DNA microarray analysis, quantitative reverse transcription polymerase chain reaction ( $Q-P C R)$, and fluorescent immunocytochemistry. DNA microarray analysis was performed by Genomic Tree Co. (Daejeon, Korea) using Agilent human whole-genome $4 \times 44 \mathrm{~K}$ chips (Santa Clara, CA). DNA microarray analysis was conducted using a previously published method (19). SCC-9 cells were treated with or without 4-HR $(10 \mu \mathrm{g} / \mathrm{ml})$ for $12 \mathrm{~h}$, and total RNA was then extracted from the cells using TRI Reagent ${ }^{\circledR}$ as recommended by the manufacturer (Molecular Research Center Inc., Cincinnati, OH).

Keratin 10 and involucrin antibodies were purchased from Santa Cruz Biotechnology for fluorescence immunocytochemistry analysis. Fluorescence immunocytochemistry was conducted using a previously published method (17). 4',6-diamidino-2-phenylindole (DAPI) was used to stain nuclei.

For the Q-PCR analysis, SCC-9 cells were treated for $12 \mathrm{~h}$ with $0,1,5$ or $10 \mu \mathrm{g} / \mathrm{ml} 4-\mathrm{HR}$. Total RNA $(1 \mu \mathrm{g})$ was used as template for first-strand DNA synthesis using the ImProm-II Reverse Transcription System (Promega, Madison, WI, USA). The RT-PCR protocol and primers used to amplify involucrin and keratin 10 were published previously $(20,21)$.

\section{Results}

4-HR decreased E2F2, E2F3, and Sp3 expression and increased Sp1 expression. In previous studies, we found that 4-HR has antitumor effects on several cancer cell lines $(17,18)$. In this study, the anti-proliferative effect of 4-HR on SCC-9 cells observed in continuous RT-CES-based assays (Fig. 1). The growth of the SCC-9 cells slowed following the addition of 4-HR. The addition of $10 \mu \mathrm{g} / \mathrm{ml} 4-\mathrm{HR}$ induced a prominent inhibitory effect, as determined by the observed decline in the growth curve (yellow) (Fig. 1). The 4-HR induced growth inhibition was dosedependent.

To identify the target genes of 4-HR, a DNA microarray comparing 4-HR treated and untreated cells was performed, and differentially expressed genes were selected for further analysis 
Table I. The results of DNA microarray. ${ }^{\mathrm{a}}$

\begin{tabular}{llc}
\hline TITLE & GenBank & Fold-ratio \\
\hline E2F2 & NM_004091 & 0.428 \\
E2F3 & NM_001949 & 0.507 \\
Sp1 & NM_138473 & 1.498 \\
Sp3 & BX648857 & 0.464 \\
Involucrin & NM_005547 & 3.176 \\
Keratin 10 & NM_000421 & 3.213 \\
Keratin 13 & NM_002274 & 2.718 \\
Keratin 14 & NM_000526 & 2.823 \\
Keratin 16 & NM_005557 & 2.635 \\
Keratin 17 & NM_000422 & 2.951 \\
Keratin 18 & NM_000224 & 2.806 \\
Keratin 19 & NM_002276 & 2.841 \\
\hline
\end{tabular}

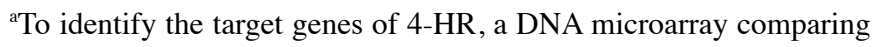
4-HR treated and untreated cells was performed.

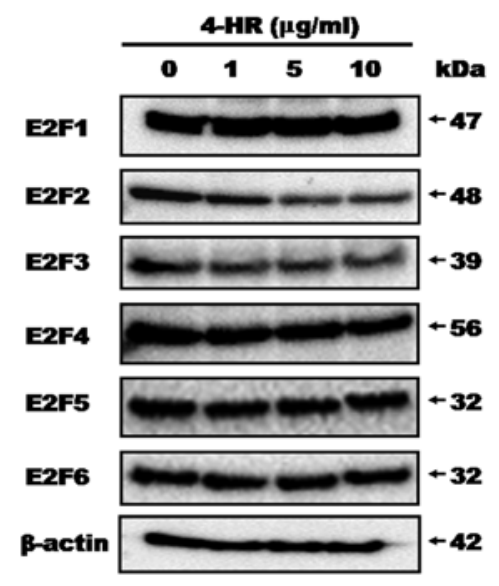

Figure 2. Western blot analyses of E2F family proteins. Western blot analyses of whole cell lysates showed that E2F2 and E2F3 protein expression significantly decreased with $4-\mathrm{HR}$ treatment.

(Table I). As shown in Fig. 1 and Table I, 4-HR decreased the levels of E2F2 and E2F3 expression. As 4-HR concentrations increased from 1 to $10 \mu \mathrm{g} / \mathrm{ml}, \mathrm{E} 2 \mathrm{~F} 2$ expression was decreased (Fig. 2). The level of E2F3 expression was also decreased in 4-HR treated cells compared to the untreated control (Fig. 2). There was no significant difference in the expression of any other E2F transcription factors; E2F1, E2F4, E2F5, and E2F6 expression were all similar in the 4-HR treated cells and the untreated control cells.

With respect to $\mathrm{Sp}$ transcription factors, $\mathrm{Sp} 1$ expression was increased by 4-HR treatment (Fig. 3A). However, Sp3 expression was decreased. DNA microarray analysis also demonstrated a decreased in the level of Sp3 expression (Table I). As a result, the $\mathrm{Sp1} / \mathrm{Sp} 3$ ratio was increased upon 4-HR treatment in a dosedependent manner. The Sp1/Sp3 ratios were 0.88, 1.72, 2.70 and 19.95 in the untreated control and in cells treated with 1, 5 and $10 \mu \mathrm{g} / \mathrm{ml} 4-\mathrm{HR}$, respectively (Fig. 3B). These results indicate that $4-\mathrm{HR}$ regulates E2F2 and E2F3 expression and the Sp1/Sp3 expression ratio in SCC-9 cells.
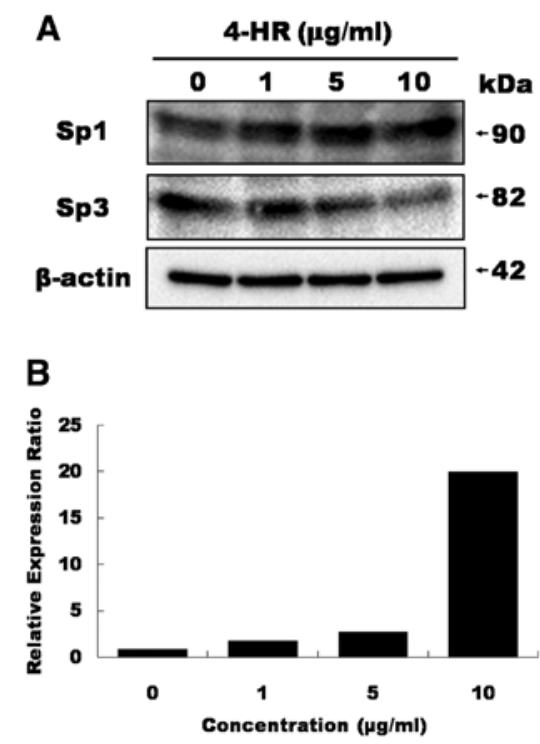

Figure 3. (A) Western blot analyses of Sp1 and Sp3. Western blot analysis for nuclear extraction showed that $\mathrm{Sp} 3$ expression significantly decreased upon 4-HR treatment, while Sp1 expression increased. (B) The relative expression ratio of $\mathrm{Sp} 1$ to $\mathrm{Sp} 3$. The $\mathrm{Sp} 1 / \mathrm{Sp} 3$ ratios were $0.88,1.72,2.70$ and 19.95 in cells treated with $0,1,5$ and $10 \mu \mathrm{g} / \mathrm{ml} 4-\mathrm{HR}$, respectively.
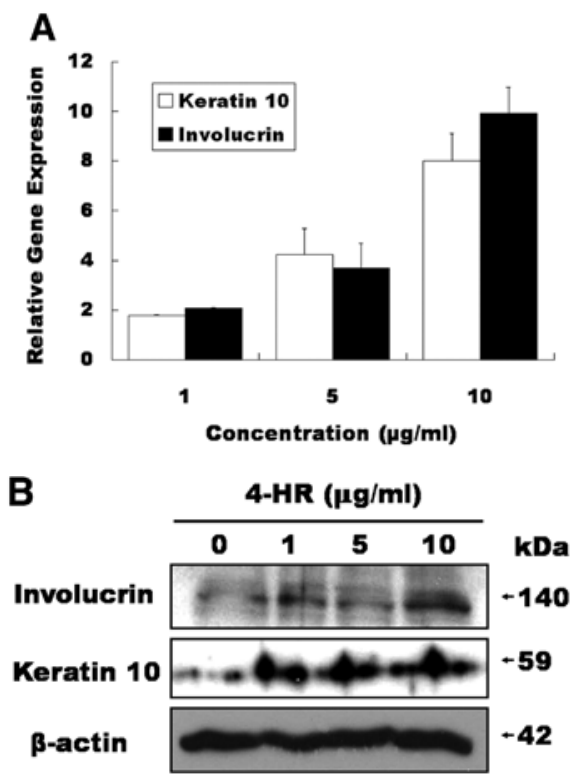

Figure 4. The expression of epithelial cell differentiation markers (keratin 10 and involucrin), selected by DNA microarray analysis, was confirmed by quantitative RT-PCR and Western blot analysis. (A) Quantitative RT-PCR analysis demonstrated that the relative mRNA levels of keratin 10 and involucrin were considerably higher in the 4-HR-treated group $(10 \mu \mathrm{g} / \mathrm{ml})$ compared with the control. Each value was normalized to GAPDH. (B) Keratin 10 and involucrin protein levels increased according to the concentrations of 4-HR. The level of $\beta$-actin was shown as a loading control.

4-HR accelerates SCC-9 cell differentiation. We also investigated genes that were involved in the epithelial cell differentiation, as identified by DNA microarray analyses. DNA microarray analysis revealed that keratins and involucrin exhibited significantly higher expression at $12 \mathrm{~h}$ after $10 \mu \mathrm{g} / \mathrm{ml}$ of 4-HR administration (Table I). The increased expression of keratin 10 and involucrin mRNA was confirmed by RT-PCR (Fig. 4A). Increased expres- 


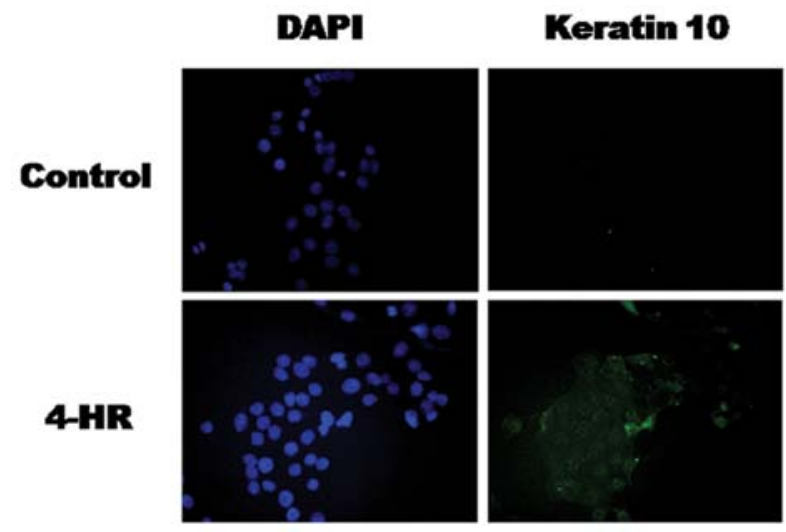

Figure 5. Fluorescent immunocytochemical analyses of the expression of keratin 10 . The level of keratin 10 expression was higher in cells treated with 4-HR than in untreated controls (bars, $20 \mu \mathrm{m}$ ).

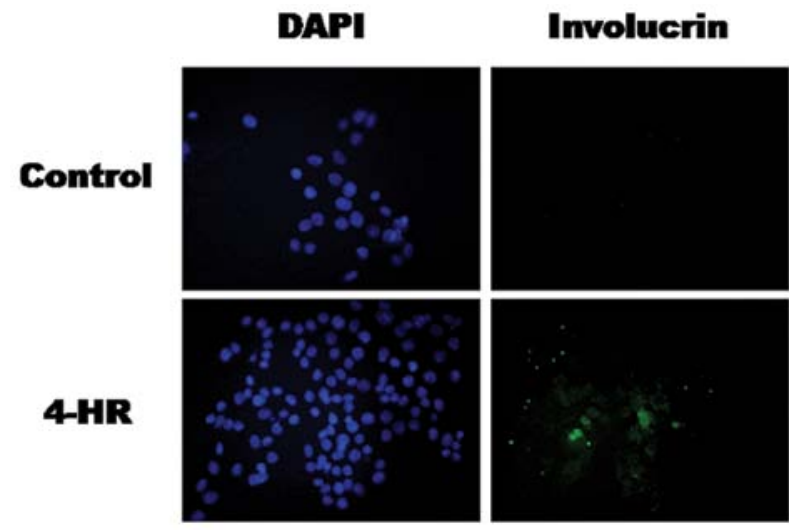

Figure 6. Fluorescent immunocytochemical analyses of the expression of involucrin. The level of involucrin expression was higher in cells treated with 4-HR than in untreated controls (bars, $20 \mu \mathrm{m}$ ).

sion of the keratin 10 and involucrin proteins was confirmed by western blot analysis (Fig. 4B) and immunocytochemistry (Figs. 5 and 6). These results indicate that 4-HR stimulates the expression of differentiation marker genes in SCC-9 cells.

\section{Discussion}

In this study, we demonstrated that 4-HR suppressed growth (Fig. 1) and stimulated the expression of differentiation marker genes in SCC-9 cells (Fig. 4). The 4-HR-induced SCC-9 cell differentiation was mediated by the E2F signaling pathway. E2F2, E2F3, and Sp3 were suppressed by 4-HR (Figs. 2 and 3). The level of Spl expression was increased by $4-\mathrm{HR}$ treatment (Fig. 3). Indeed, 4-HR increased the expression of the epithelial cell differentiation markers keratin 10 and involucrin. Taken together, these data show that 4-HR induced SCC-9 cell differentiation through the downregulation of $\mathrm{E} 2 \mathrm{~F} 2$ and upregulation of $\mathrm{Sp} 1 / \mathrm{Sp} 3$ ratio.

Senescence is defined as a permanent cell cycle arrest induced by oncogene expression and it is an effective anti-cancer mechanism in vivo (22-24). Therefore, treatments aiming to inhibit the uncontrolled growth of cancer cells while simultaneously restoring their normal differentiation program would be invaluable (1). To develop such 'differentiation therapies', it is important to find candidate drugs that can control the E2F signaling pathway.

Squamous epithelial cell differentiation is controlled by Sp1 and E2F (25). Sp1 can act as a differentiation activator and its activity is regulated by E2F (1). E2F1, E2F2, and E2F3 are expressed highly in human osteosarcoma cells, and a large number of genes with known role in development and differentiation are consequently deregulated (26). However, the inhibition of E2F alone or the introduction of Sp1 alone is not sufficient to reinstate squamous differentiation (1). In the present study, 4-HR was able to suppress E2F2 and E2F3 expression (Fig. 2). At the same time, $\mathrm{Sp} 1$ expression was increased, and $\mathrm{Sp} 3$ expression was decreased (Fig. 3A). The Sp1 to Sp3 ratio is important in the regulation of cell differentiation, and the increase in this ratio observed upon 4-HR treatment (Fig. 3) is consistent with the activation of tumor cell differentiation (1).

In keratinocytes, E2F inhibition is a prerequisite for differentiation, and E2Fs 1-5 are all able to suppress differentiation-specific markers $(3,4)$. E2F suppresses multiple markers of squamous cell differentiation $(3,27)$. E2F is also able to suppress the differentiation induced by multiple stimuli $(3,27)$. Thus, E2F has the capacity to act as an important regulator of squamous cell differentiation. In this study, the increased expression of differentiation markers such as keratin 10 and involucrin was due in part to the combination of the inhibition of E2F2 and E2F3 and the upregulation of the Sp1/Sp3 ratio by 4-HR (Fig. 4). However, these observations were made at the cellular level, and it will be important to validate these results in vivo. To this end, systemic administration of 4-HR in xenografted mice has been performed, and the expression of differentiation markers in the xenografted tumor mass is currently under investigation (data not shown).

In conclusion, 4-HR stimulated the differentiation of SCC-9 cells, potentially through the suppression of the E2F signaling pathway.

\section{Acknowledgements}

This study was supported by a grant from the Next-Generation BioGreen21 Program (Center for Nutraceutical \& Pharmaceutical Materials no. PJ009051), Rural Development Administration, Republic of Korea.

\section{References}

1. Wong CF, Barnes LM, Dahler AL, et al: E2F suppression and $\mathrm{Spl}$ overexpression are sufficient to induce the differentiationspecific marker, transglutaminase type 1 , in a squamous cell carcinoma cell line. Oncogene 24: 3525-3534, 2005.

2. Bruckman KC, Schönleben F, Qiu W, Woo VL and Su GH: Mutational analyses of the BRAF, KRAS, and PIK3CA genes in oral squamous cell carcinoma. Oral Surg Oral Med Oral Pathol Oral Radiol Endod 110: 632-637, 2010.

3. Wong CF, Barnes LM, Dahler AL, et al: E2F modulates keratinocyte squamous differentiation: implications for E2F inhibition in squamous cell carcinoma. J Biol Chem 278: 28516-28522, 2003.

4. Wong CF, Barnes LM, Smith L, Popa C, Serewko-Auret MM and Saunders N: E2F6: a member of the E2F family that does not modulate squamous differentiation. Biochem Biophys Res Commun 324: 497-503, 2004.

5. Polyak K, Kato JY, Solomon MJ, et al: $27^{\mathrm{Kip} 1}$, a cyclin-Cdk inhibitor, links transforming growth factor-beta and contact inhibition to cell cycle arrest. J Gene Dev 8: 9-22, 1994. 
6. DeGregori J, Kowalik T and Nevins JR: Cellular targets for activation by the E2F1 transcription factor include DNA synthesis- and G1/S-regulatory genes. Mol Cell Biol 15: 4215-4224, 1995.

7. DeGregori J, Leone G, Miron A, Jakoi L and Nevins JR: Distinct roles for E2F proteins in cell growth control and apoptosis. Proc Natl Acad Sci USA 94: 7245-7250, 1997.

8. Wu L, Timmers C, Maiti B, et al: The E2F1-3 transcription factors are essential for cellular proliferation. Nature 414: 457-462, 2001

9. Wu Z, Zheng S and Yu Q: The E2F family and the role of E2F1 in apoptosis. Int J Biochem Cell Biol 41: 2389-2397, 2009.

10. Wang J, Helin K, Jin P and Nadal-Ginard B: Inhibition of in vitro myogenic differentiation by cellular transcription factor E2F1. Cell Growth Differ 6: 1299-1306, 1995.

11. Guy CT, Zhou W, Kaufman S and Robinson MO: E2F-1 blocks terminal differentiation and causes proliferation in transgenic megakaryocytes. Mol Cell Biol 16: 685-693, 1996.

12. Fajas L, Landsberg RL, Huss-Garcia Y, Sardet C, Lees JA and Auwerx J: E2Fs regulate adipocyte differentiation. Dev Cell 3 39-49, 2002

13. Kozubek A and Tyman JHP: Resorcinolic lipids, the natural nonisoprenoid phenolic amphiphiles and their biological activity. Chem Rev 99: 1-25, 1999.

14. Mutoh M, Takahashi M, Fukuda K, et al: Suppression of cyclooxygenase-2 promoter-dependent transcription activity in colon cancer cells by chemopreventive agents with a resorcin-type structure. Carcinogenesis 21: 959-963, 2000.

15. Hasegawa R, Furukawa F, Toyoda K, et al: Inhibitory effect of antioxidants on N-bis(2-hydroxypropyl)nitrosamine-induced lung carcinogenesis in rats. Jpn J Cancer Res 81: 871-877, 1990.

16. Maruyama H,Amamura T,Nakae D, et al: Effect of catechol and its analogs on pancreatic carcinogenesis initiated by $N$-nitrosobis $(2-$ oxopropyl)amine in Syrian hamsters. Carcinogenesis 12 1331-1334, 1991.

17. Kim SG, Jeong JH, Park YW, et al: 4-Hexylresorcinol inhibits transglutaminase-2 activity and has synergistic effects along with cisplatin in KB cells. Oncol Rep 25: 1597-1602, 2011.
18. Kim SG, Lee SW, Park YW, Jeong JH and Choi JY: 4-hexylresorcinol inhibits NF- $\kappa$ B phosphorylation and has a synergistic effect with cisplatin in KB cells. Oncol Rep 26: 1527-1532, 2011.

19. Kim JY, Choi JY, Jeong JH, et al: Low molecular weight silk fibroin increases alkaline phosphatase and type I collagen expression in MG63 cells. BMB Rep 43: 52-56, 2010.

20. Trollinger DR, Cascio WE and Lemasters JJ: Selective loading of Rhod 2 into mitochondria shows mitochondrial $\mathrm{Ca}^{2+}$ transients during the contractile cycle in adult rabbit cardiac myocytes. Biochem Biophy Res Commun 236: 738-742, 1997.

21. Wagenblast J, Baghi M, Arnoldner C, et al: Effect of bortezomib and cetuximab in EGF-stimulated HNSCC. Anticancer Res 28: 2239-2243, 2008

22. Braig M, Lee S, Loddenkemper C, et al: Oncogene-induced senescence as an initial barrier in lymphoma development. Nature 436: 660-665, 2005.

23. Chen Z, Trotman LC, Shaffer D, et al: Crucial role of p53-dependent cellular senescence in suppression of Pten-deficient tumorigenesis. Nature 436: 725-730, 2005.

24. Michaloglou C, Vredeveld LC, SoengasMS, et al: BRAFE600associated senescence-like cell cycle arrest of human naevi. Nature 436: 720-724, 2005

25. Hazar-Rethinam M, Cameron SR, Dahler AL, et al: Loss of E2F7 expression is an early event in squamous differentiation and causes derepression of the key differentiation activator Sp1. J Invest Dermatol 131: 1077-1084, 2011.

26. Muller H, Bracken AP, Vernell R, et al: E2Fs regulate the expression of genes involved in differentiation, development, proliferation, and apoptosis. Genes Dev 15: 267-285, 2001.

27. Dicker AJ, Popa C, Dahler AL, et al: E2F-1 induces proliferationspecific genes and suppresses squamous differentiation-specific genes in human epidermal keratinocytes. Oncogene 19: 2887-2894, 2000. 